\title{
Neutrino Factory Accelerator R\&D: Status and Priorities
}

\author{
M. S. Zisman ${ }^{\dagger}$ \\ Accelerator \& Fusion Research Division, Lawrence Berkeley National Laboratory \\ One Cyclotron Road, Berkeley, CA 94720 USA
}

This paper summarizes the status of worldwide Neutrino Factory R\&D efforts. Activities are categorized as simulations, component development, and system tests. An indication of R\&D tasks that remain to be accomplished is also given.

\section{INTRODUCTION]}

Construction of a muon-based Neutrino Factory will be a challenging endeavor. Muons have a short lifetime $(2.2 \mu \mathrm{s}$ at rest), which means that all beam manipulations must be done quickly. In addition, muons are a tertiary beam $(\mathrm{p} \rightarrow \pi \rightarrow \mu)$, which means they are created with low intensity and with a large $6 \mathrm{D}$ phase space. The low intensity implies a very high proton beam power and hence a requirement for a high-power target. The large energy spread, large transverse beam sizes, and large angular spread do not lend themselves well to transport in standard accelerator components.

We must successfully address these challenges to make the Neutrino Factory a worthwhile option for high-energy physics. Required solutions are well beyond those required for "standard" accelerator systems. Developing and demonstrating such solutions is the aim of the global R\&D effort described here. This R\&D program will thus allow the high-energy physics community to make an informed decision about the desirability of a Neutrino Factory by specifying its expected performance, its technical feasibility and degree of risk, and its approximate cost.

In what follows, I give an overview of the global Neutrino Factory R\&D program. Of necessity, this description will be brief and incomplete. I also give my views on what work will be needed to reach the stage of being ready to produce a Conceptual Design Report and cost estimate for a Neutrino Factory.

\footnotetext{
${ }^{\dagger}$ Work supported by the Director, Office of Science, High Energy Physics, U. S. Department of Energy under Contract No. DE-AC02-05CH11231.
}

\section{R\&D MISSION AND APPROACHES}

The mission of the Neutrino Factory R\&D effort is to develop conceptual solutions to produce, condition, accelerate, and store intense muon beams, to demonstrate technical viability of critical components, and to make an estimate of the overall cost of the facility. Three main approaches are followed in the Neutrino Factory R\&D program: simulations; component development; and system tests. Conceptual solutions from the R\&D program must first be seamlessly integrated into an overall facility concept, after which estimates of performance (in terms of neutrinos per year) and cost can be obtained. To be useful, the cost estimate must be based on a study of alternatives sufficient to identify the most cost-effective approaches.

The task of the simulation group is to develop and validate the simulation codes and then apply them to design studies of individual subsystems and the overall facility. The earlier feasibility studies [1-4] and the recently launched International Scoping Study (ISS) [5] are examples of this work. Component development involves the fabrication and laboratory testing of critical devices. System tests are used to validate, via engineering demonstrations, the performance of key subsystems (e.g., the target or the cooling channel) to ensure that they behave as predicted.

\section{PARTICIPANTS}

The evolution of the Neutrino Factory R\&D effort into an international program began informally, via sharing of information among the groups. This was very successful in avoiding unnecessary duplication. 
The international Neutrino Factory workshops [6] have played an important role in this evolution.

Recently, jointly coordinated programs have become more common. This coordination takes place at the working level, rather than being dictated by funding agencies or laboratory management. Such grass-roots collaboration is by far the most effective kind, as it is driven by scientific goals. Examples of such international activities include MICE, nTOF11, the ISS, the APS Neutrino Physics Study, and the EMMA FFAG collaboration.

\section{R\&D STATUS}

\subsection{Simulations}

To date, there have been four Neutrino Factory feasibility studies, two in the U.S. [1, 2], one in Japan [3], and one in Europe [4]. In addition, U.S. Feasibility Study II was recently updated [7] during the course of the American Physical Society Neutrino Physics Study. We refer to this update as "Study IIa." The Neutrino Factory design activity will continue in the next year with the ISS.

Study IIa was able to maintain the performance of Study II while providing the possibility of keeping both muon signs simultaneously. Moreover, improvements in the design led to a substantially reduced cost estimate-roughly two-thirds that of Study II. The hardware cost of a Neutrino Factory is now estimated to be approximately $\$ 1 \mathrm{~B}$.

Although several models of scaling FFAG rings have been successfully built and tested in Japan [8], the newer non-scaling FFAG concept has yet to be tested. Substantial progress has been made recently in the design of non-scaling FFAG rings, and there is a proposal [9] to build an electron model of such a device at Daresbury Laboratory. Figure 1 shows the lattice of the $15.9 \mathrm{~m}$ circumference EMMA ring.

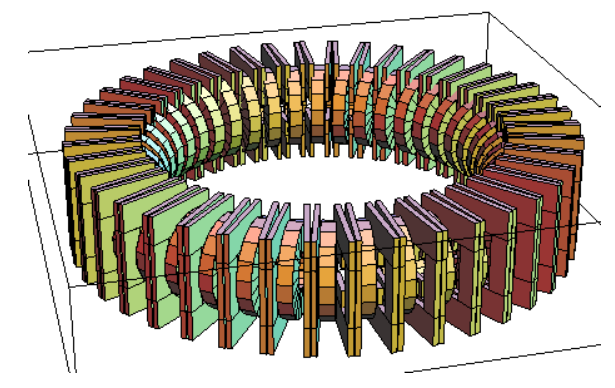

Figure 1. Lattice of test model FFAG ring (EMMA).
Designs of cooling rings, which potentially provide $6 \mathrm{D}$ cooling, are also under study $[10,11]$. Figure 2 shows a possible layout of such a ring. At this time, the main focus of the design effort is to develop viable ring concepts that show adequate dynamic aperture in tracking studies.

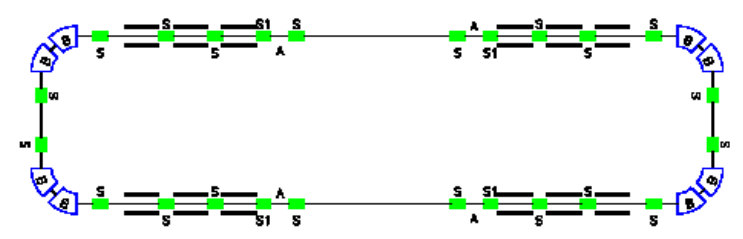

Figure 2. Example of cooling ring design with both dipoles and solenoids [11].

Considerable simulation activity has gone into the study of target behavior, both for solid and liquid-jet targets. In Europe, the emphasis has been on solid targets. Studies of the beam-heating effects in a solid target indicate [12] that spacing the bunches by more than a few microseconds is enough to decouple their thermal effect in the target. Simulations of mercury behavior when hit with a proton beam [13] bear remarkable similarity to the experimental results, as shown in Fig. 3.

\subsection{Component Development}

RF cavities are a key technology for a Neutrino Factory, and considerable effort has gone into their development. The Study II cooling channel requires high-gradient 201-MHz cavities operating in a $2-\mathrm{T}$ magnetic field. A prototype cavity has recently been
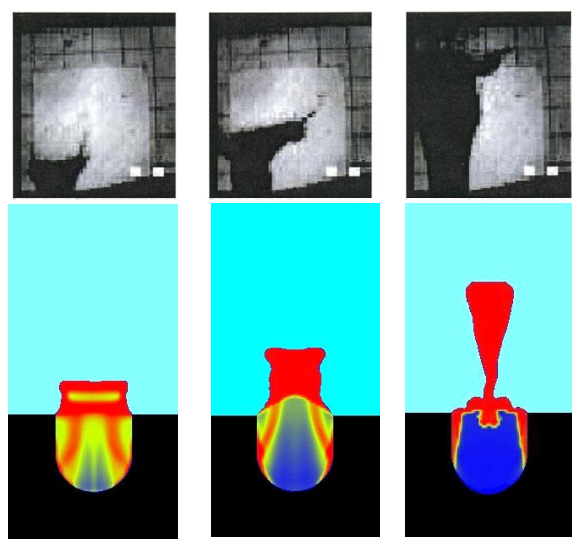

Figure 3. Observed behavior of free mercury hit by proton beam (top) compared with simulations (bottom) [13]. 
completed by the Neutrino Factory and Muon Collider Collaboration (NFMCC) [14]. This cavity will have TiN-coated Be windows and is designed to have a ratio of peak surface field to accelerating field close to unity. Figure 4 shows the cavity undergoing low-power testing at Jlab.

Superconducting RF cavities are also needed for a Neutrino Factory facility. They serve in the acceleration system, where magnetic fields of the type used for the cooling channel can be avoided. They are also used for the proton driver in the CERN scenario, which is based on the $3.5 \mathrm{GeV}$ SPL [15].

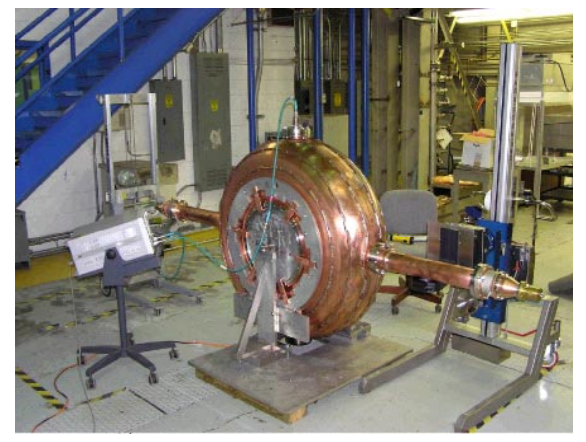

Figure 4. 201-MHz prototype RF cavity.

Because of its low $Z$ and large radiation length, liquid hydrogen $\left(\mathrm{LH}_{2}\right)$ is an ideal absorber material for a cooling channel. However, its other aspects, including flammability and cryogenic temperature, make it a challenge to use. An absorber will be tested under realistic operating conditions in the MICE [16] cooling channel, though not at the large absorbed power that would be representative of an actual Neutrino Factory.

Target components are also challenging and require development. A continuous Hg-jet system, being studied at Princeton, is shown in Fig. 5. A more sophisticated version of this system will be incorporated in the nTOF11 experiment at CERN [17]. Compared with a high-field solenoid, a horn has the advantages of being simple to fabricate and relatively inexpensive [18]. However, it has a short lifetime, which implies frequent replacement, and it only focuses one muon sign at a time. The main R\&D issues for a horn are radiation hardness, mechanical strength when pulsing, and overall reliability. These aspects are best evaluated by building and testing prototypes.

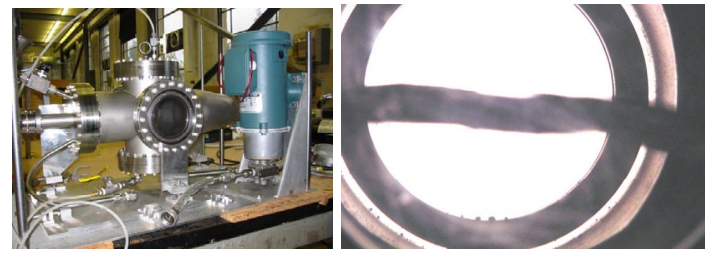

Figure 5. (Left) Hg-jet apparatus; (right) photo of $\mathrm{Hg}$ jet on a good day.

\subsection{System Tests}

System tests are needed to confirm the performance of integrated systems, that is, to demonstrate that the technologies operate as a functional unit. As these tests tend to be relatively expensive, we must be selective in choosing which to pursue. The activities currently under way include MICE, nTOF11, PRISM, and FETS. Most of these are being carried out as international ventures, with the additional virtue of being good team-building exercises.

MICE, the Muon Ionization Cooling Experiment, is an approved experiment at Rutherford Appleton Laboratory [16]. The main challenges of MICE are operating high-gradient RF cavities in a high magnetic field and with field terminations (windows), safely operating $\mathrm{LH}_{2}$ absorbers with very thin windows, and integrating the cooling channel components while maintaining operational functionality. There is also the experimental challenge of measuring a relatively small change in emittance, about $10 \%$, with a precision of $10^{-3}$.

In December 2004, MICE Phase I passed its Gateway review in the UK, thus releasing $£ 9.7 \mathrm{M}$ in funding. Funds from international partners (Japan, Switzerland, U.S.) are also being made available. First beam is anticipated in April 2007. A layout of the experiment is shown in Fig. 6.

The nTOF11 experiment [17], proposed by an international group from BNL, CERN, KEK, ORNL, Princeton, and RAL, has recently been approved at CERN. It will test the performance of a mercury-jet target in a 15-T solenoid (see Fig. 7). A $24-\mathrm{GeV}$ proton beam from the PS will provide a power deposition $(180 \mathrm{~J} / \mathrm{g})$ representative of that seen by a Neutrino Factory target bombarded with a $4 \mathrm{MW}$ proton beam. The experiment is scheduled for April 2007. This experiment is a follow-on effort to BNL experiment E951 [19], in which a mercuryjet target was exposed to a $24-\mathrm{GeV}$ proton beam from the AGS in the absence of a magnetic field. 


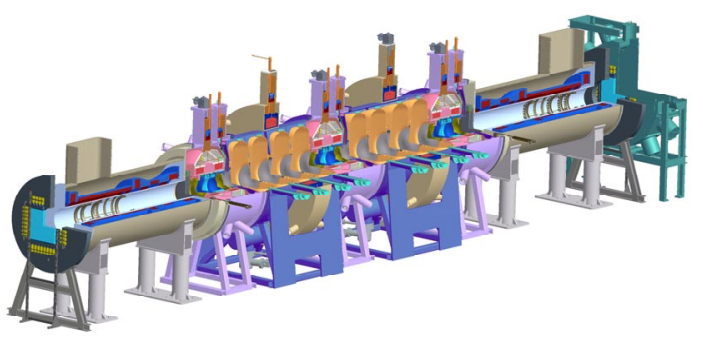

Figure 6. Cutaway 3D view of MICE layout. Upstream and downstream solenoids house the trackers; the central section includes one cell of the Study II cooling channel.
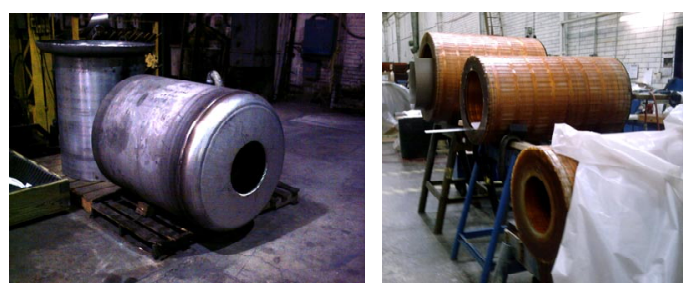

Figure 7. (Left) cryostat for 15-T solenoid; (right) coils for magnet. Coils are nested to produce the desired field.

PRISM [20] will demonstrate the phase rotation of a slow muon beam with a scaling FFAG. This experiment at Osaka University is not motivated by accelerator physics per se, but by using a slow muon beam having a low energy spread. Nonetheless it will demonstrate this technology for producing a high-quality muon beam, and thus serves to validate the accelerator concept. The first phase of the experiment, building the ring (see Fig. 8), is approved and will be completed in 2007. The hope is to obtain funding to complete the full experimental setup by 2009 .

There are front-end test stands (FETS) being developed at CERN [21] and at RAL [22], and equivalent efforts at J-PARC and likely soon at Fermilab. There are many interested "customers" for such devices, which can serve as a source for producing neutrons, neutrinos, or rare isotopes, and could play a role in nuclear-waste transmutation. Hopefully, synergies among the various customers will be exploited where possible. Studies involve optimization of the $\mathrm{H}^{-}$ion source, the chopper, and the buncher. Efficient chopping and bunching of the beam are critical with a high-power proton driver, as lost beam could easily render the accelerator structure too radioactive for hands-on maintenance.

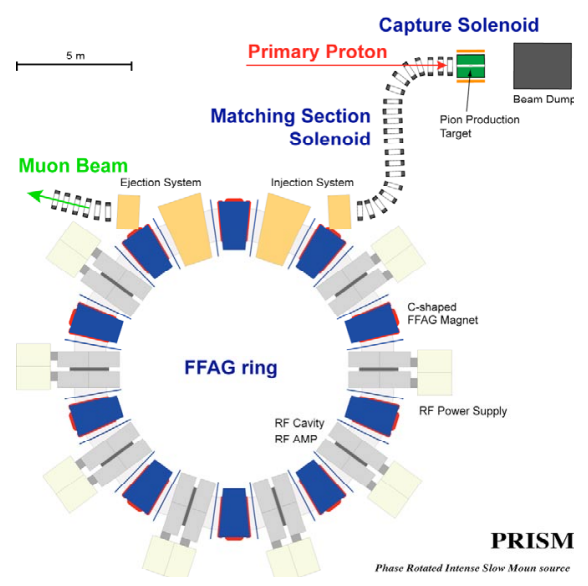

Figure 8. Diagram of PRISM ring and injection system.

\section{REMAINING R\&D ISSUES}

In general, the list of topics being studied is appropriate. There is evidence of good coordination and communication among the various $\mathrm{R} \& \mathrm{D}$ groups. However, the need for multiple FETS R\&D efforts has not been well articulated.

Priorities for the R\&D program should be to:

- complete current component R\&D

- bring system studies to fruition

- embark on the ISS

There are a few items that have not received adequate attention in the past and need to be studied more carefully. These include the design of the storage ring and its magnets, the development of an optimized acceleration scheme, the evaluation of alternative absorber materials, and the testing of a solid target in a realistic Neutrino Factory configuration (i.e., the solid-target counterpart of the nTOF11 experiment).

\section{ISSUES TO STUDY DURING THE ISS}

The goal of the ISS is to narrow the range of options, ideally to a single choice, in preparation for a later World Design Study. To accomplish this, a number of decisions must be reached; hopefully, there will be an international consensus on these. (The required decisions are discussed in Ref. [23].) In practice, optimizing the design and reducing the number of options require information on relative costs. It is thus very important that there be some engineering involvement in the ISS, and that an 
agreed-upon cost model be identified. Both the accelerator and detector should be included in the optimization process.

\section{SUMMARY}

Neutrino Factory design progress has been excellent in recent years. The estimated performance of the facility has improved and the estimated cost has declined. The R\&D program has been carried out as an international effort, and this has gone very well. Such international cooperation must continue. Another very successful feature of the R\&D effort is the collaboration between accelerator and particle physicists. Both these aspects have been real strengths, and they set an example for other international collaborations to follow.

The goal of the upcoming ISS is to narrow the technical options. Converging on specific choices is always difficult, but our international collaboration is strong enough to accomplish this as a team. This will be a necessary step toward our ultimate goalconvincing a laboratory somewhere to identify the Neutrino Factory as its next project.

\section{REFERENCES}

[1] N. Holtkamp, D. Finley (eds.), A Feasibility Study of a Neutrino Factory Based on a Muon Storage Ring, August 2000; see http://www.fnal.gov/projects/muon_collider/nu /study/report/machine report/.

[2] S. Ozaki, R. Palmer, M. S. Zisman, J. Gallardo (eds.), Feasibility Study II of a Muon-Based Neutrino Source, BNL-52623, June 2001; see http://www.cap.bnl.gov/mumu/studyii/final_dr aft/The-Report.pdf.

[3] Y. Kuno and Y. Mori (eds.), A Feasibility Study of a Neutrino Factory in Japan, http://www-prism.kek.jp/nufactj/nufactj.pdf.

[4] P. Gruber (ed.), The Study of a European Neutrino Factory Complex, see http://slap.web.cern.ch/slap/NuFact/NuFact/nfl 22.pdf.

[5] http://dpnc.unige.ch/users/blondel/ISSatCERN .htm

[6] http://www.lnf.infn.it/conference/2005/nufact $05 /$, and references to previous workshops contained therein.

[7] C. Albright, et al., Neutrino Factory and Beta Beam Experiments and Development, BNL72369-2004, FNAL-TM-2259, LBNL-55478; http://www.aps.org/neutrino/loader.cfm?url=/c ommonspot/security/getfile.cfm\&PageID $=587$ 66

[8] S. Machida et al., Proc. of EPAC 2004, Lucerne, Switzerland, July 5-9, 2004, p. 2643.

[9] C. Johnstone, Hardware Development for EMMA, these proceedings; http://hepunx.rl.ac. uk/uknf/wp1/emodel/nest/NEST-FFAGproposal-v6.doc.

[10] S. Kahn et al., Tetra Cooling Ring, AIP Conf. Proc. 721, 2004, p. 387; Y. Fukui, D. Cline, A. Garren, A Muon Ring Cooler with Lithium Lenses, AIP Conf. Proc. 721, 2004, p. 395.

[11] S. Brooks et al., Features of a Muon Cooling Ring for a Neutrino Factory, AIP Conf. Proc. 721, 2004, p. 399.

[12] J.R.J. Bennett, Solid Target Studies in the UK, these proceedings.

[13] R. Samulyak, Numerical Simulation of Hydroand Magnetohydro-dynamic Processes in the Muon Collider Target, Lecture Notes in Computer Science 2331, 391 (2002).

[14] D. Li et al., Proc. of PAC 2003, Portland, OR, May 12-16, 2003, p. 1243.

[15] R. Garoby, The SPL at CERN, Proc.of Workshop on High Intensity High Brightness Hadron Beams, Bensheim, Darmstadt, Germany, October 18-24, 2004, p. 239.

[16] Y. Torun, MICE Status, AIP Conf. Proc. $\underline{721}$, 2004, p. 106.

[17] J.R.J. Bennett et al., Studies of a Target System for a $4 \mathrm{MW}, 24 \mathrm{GeV}$ Proton Beam, http://www.pparc.ac.uk/Rs/Pp/Sp/TT2A propo sal.pdf.

[18] S. Gilardoni et al., Horn R\&D for 2002-2003, AIP Conf. Proc. 721,2004 , p. 334.

[19] J. Alessi et al., An R\&D Program for Targetry at a Muon Collider, proposal to the BNL AGS, September 1998.

[20] The PRISM Project-A Muon Source of the World's Highest Brightness by Phase Rotation, LOI for Nuclear and Particle Physics Experiments at the J-PARC, 2003.

[21] R. Garoby, Proton Driver: Prospects in Europe, these proceedings.

[22] J. Pozimski, Progress at the RAL Front-End Test Stand, these proceedings.

[23] M. Zisman, Review of U.S. Neutrino Factory Studies, these proceedings. 